\title{
Treatment of lumbar synovial cysts using minimally invasive surgical techniques
}

\author{
Nouzhan Sehati, M.D., Larky T. Khoo, M.D., and Langston T. Holly, M.D. \\ Division of Neurosurgery, David Geffen University of California at Los Angeles School of Medicine, \\ Los Angeles, California
}

\begin{abstract}
Object. Lumbar synovial cysts are a potential cause of radiculopathy and back pain, and the definitive treatment is the complete excision of the cyst. This report summarizes the authors' preliminary clinical experience with the minimally invasive resection of lumbar synovial cysts.

Methods. Nineteen patients (nine men and 10 women) with symptomatic synovial cysts underwent minimally invasive resection. The mean patient age was 64 years of age (range $43-80$ years). The presenting symptom was radiculopathy in 16 patients, low-back pain in two, and lower-extremity weakness in one. There were 16 cases of a cyst located at the L4-5 level, two at L3-4, and one at L5-S1. The mean cyst diameter was $13.7 \mathrm{~mm}$ (range 3-30 mm).

The mean follow-up time was 16 months (range 4-29 months). Clinical outcomes were graded, based on the Macnab modified criteria, as excellent, good, fair, or poor. Eighteen patients (95\% of cases) reported either excellent (10 patients) or good (eight patients) results, and a fair result was reported by one patient (5\% of cases). The mean operative time was 158 minutes (range 75-270 minutes), and the average intraoperative blood loss was $31 \mathrm{ml}$ (range 10-100 $\mathrm{ml}$ ). Two patients had intraoperative dural tears that resulted in cerebrospinal fluid leaks that resolved following primary closure.

Conclusions. Synovial cysts can be safely and effectively treated using minimally invasive surgical techniques. Long-term follow up is required to determine whether this approach results in less need for fusion than conventional surgical approaches.
\end{abstract}

KEY WORDS • minimally invasive surgery - spine • synovial cyst

\section{$\mathrm{L}$} UMBAR spinal synovial cysts are thought to develop as a consequence of osteoarthritic changes of the facet joints, and they can be a cause of radiculopathy and low-back pain. $6,10,15,18$ Nonoperative management including the use of nonsteroidal antiinflammatory drugs, physical therapy, and epidural steroids is the first line of treatment in patients with symptomatic synovial cysts. Excision is reserved for cysts that cause neurological deficits or intractable pain, or for cysts in patients in whom conservative therapy has failed.

Because synovial cysts are directly related to degenerated facet joints, the distinct risk of spinal instability following removal of these lesions exists. In part to address these concerns, the primary surgical treatment of lumbar synovial cysts has evolved from performing a complete laminectomy to performing a hemilaminotomy, directly over the lesion in many cases. The latest modification of this procedure involves the integration of minimally invasive techniques that were initially developed for lumbar microdiscectomy. ${ }^{4}$ Minimally invasive techniques reduce the risk of approach-related iatrogenic soft-tissue injury

Abbreviations used in this paper: $\mathrm{EBL}=$ estimated blood loss; $\mathrm{LOS}=$ length of stay; MR = magnetic resonance. and have been shown to decrease intraoperative blood loss, postoperative pain, and LOS. ${ }^{3}$

Numerous authors have previously described the excision of lumbar synovial cysts using conventional surgical methods; $1,5,8,12,14,16$ however, there are very few reports that focus on the minimally invasive technique. In this manuscript, we seek to detail our preliminary clinical experience with the minimally invasive resection of lumbar synovial cysts.

\section{Clinical Material and Methods}

\section{Patient Population}

Between July 2003 and August 2005, 19 consecutive patients with lumbar synovial cysts underwent a minimally invasive resection of the lesion at the University of $\mathrm{Ca}$ lifornia at Los Angeles Medical Center (Table 1). A retrospective review of hospital charts and MR images was performed for each patient, and all patients were included in the study. There were 10 women and nine men with a mean age of 64 years (range 43-80 years). All patients underwent 6 weeks of nonoperative management prior to surgery, which included one or more of the following modalities: nonsteroidal antiinflammatory drugs, physical therapy, and epidural steroid injections. None of the pa- 
N. Sehati, L. T. Khoo, and L. T. Holly

tients had previously undergone surgery at the level of the cyst or at adjacent spinal segments.

\section{Signs and Symptoms}

Radicular leg pain was the most common chief complaint and was encountered in 16 patients. Two patients reported primarily low-back pain, with radicular pain being a smaller component of the symptomatology; the other patient reported leg weakness as the primary problem. The radicular symptoms were located solely on the left side in nine patients, solely on the right side in eight, and bilaterally in two patients.

\section{Radiographic Imaging}

Magnetic resonance imaging was used in each case to diagnose the synovial cyst as well as to determine its location and size (Fig. 1). There were 16 cases in which the cyst was located at the L4-5 level, two at L3-4, and one at L5-S1. The cysts were located on the left side in 10 patients, on the right side in eight patients, and one patient had bilateral synovial cysts. The mean cyst diameter was $13.7 \mathrm{~mm}$ (range 3-30 mm; Fig. 2). Plain anteroposterior and lateral radiographs were obtained in all patients. Flexion-extension x-ray studies were obtained in two patients who had evidence of Grade I spondylolisthesis. There was no evidence of spinal instability in either of these patients.

\section{Operative Technique}

After induction of general anesthesia, the patient is securely positioned prone on a Wilson frame with the spine flexed to aid exposure of the intralaminar space. The patient is prepared and draped in a sterile fashion, and a spinal needle is used to identify the vertebral level of interest with the aid of fluoroscopic guidance. An 18-mm incision approximately $15 \mathrm{~mm}$ lateral to the midline is then centered on this point. A K-wire is used to penetrate the muscle and fascia, and serial dilators are passed over the wire and docked at the junction of the lamina and facet. The appropriate-length METRx tubular retractor (Medtronic Sofamor Danek, Memphis, TN) is passed over the dilators and affixed to a bed-mounted arm. Lateral fluoroscopy is again used, this time to confirm proper positioning of the tubular retractor.

With visualization provided by the operative microscope, the thin layer of soft tissue over the lamina and medial facet is removed using monopolar cautery. A curette is used to expose the inferior edge of the lamina. A Kerrison instrument and/or high-speed drill is then used to perform the hemilaminotomy and medial facetectomy. If a complete laminectomy is required, the tubular retractor can be angled more medially and the patient tilted away from the surgeon to provide a more contralateral view. The pedicle frequently serves as an excellent landmark from which to determine the extent of necessary lateral bone removal and to maintain anatomical orientation (Fig. 3 ). The ligamentum flavum is opened either cephalad or caudal to the cyst. At this point, the cyst should be carefully separated from the dura mater by using curved curettes as well as sharp dissection. The traversing nerve root should be identified. In some cases, the cyst can be resected en bloc, but commonly it will require piecemeal re-
TABLE 1

Demographic data for 19 patients with lumbar synovial cysts

\begin{tabular}{cccl}
\hline \hline Case No. & Age (yrs), Sex & Cyst Level & Main Symptom \\
\hline 1 & $73, \mathrm{M}$ & L4-5 & radicular pain \\
2 & $79, \mathrm{~F}$ & L3-4 & leg weakness \\
3 & $72, \mathrm{~F}$ & L4-5 & low-back pain \\
4 & $58, \mathrm{M}$ & L4-5 & radicular pain \\
5 & $71, \mathrm{M}$ & L4-5 & low-back pain \\
6 & $58, \mathrm{~F}$ & L4-5 & radicular pain \\
7 & $69, \mathrm{~F}$ & L4-5 & radicular pain \\
8 & $43, \mathrm{M}$ & L4-5 & radicular pain \\
9 & $61, \mathrm{M}$ & L5-S1 & radicular pain \\
10 & $59, \mathrm{~F}$ & L4-5 & radicular pain \\
11 & $51, \mathrm{M}$ & L4-5 & radicular pain \\
12 & $80, \mathrm{~F}$ & L4-5 & radicular pain \\
13 & $67, \mathrm{~F}$ & L3-4 & radicular pain \\
14 & $63, \mathrm{~F}$ & L4-5 & radicular pain \\
15 & $58, \mathrm{M}$ & L4-5 & radicular pain \\
16 & $59, \mathrm{~F}$ & L4-5 & radicular pain \\
17 & $63, \mathrm{M}$ & L4-5 & radicular pain \\
18 & $74, \mathrm{M}$ & L4-5 & radicular pain \\
19 & $51, \mathrm{~F}$ & L4-5 & radicular pain \\
\hline
\end{tabular}

moval, particularly with larger cysts. Careful dissection and establishment of the proper anatomical planes can minimize the risk of cerebrospinal fluid leakage, but in some cases dural tearing is unavoidable. Primary closure of the durotomy is technically feasible, but in most patients this only requires placement of a collagen sponge and fibrin glue, followed by 24 to 72 hours of bedrest.

\section{Results}

The mean follow-up time was 16 months (range 4-29 months). The synovial cysts were removed using a hemilaminotomy in 17 patients, and the remaining two underwent a laminectomy. Of these two patients, one had bilateral symptoms related to bilateral synovial cysts and the other had contralateral foraminal stenosis. Thirteen of the 19 patients were discharged home on the day of surgery (Table 2). Two other patients could have been discharged on the day of surgery but preferred to stay overnight because they lived a significant distance from the hospital. Two patients were discharged 48 hours after surgery; their increased stays were related to periincisional pain control. The remaining two patients had intraoperative cerebrospinal fluid leaks that required several days of bedrest, which delayed their discharge.

The patients' postoperative outcomes were graded using the Macnab modified criteria: ${ }^{13}$ excellent, complete resolution of symptoms; good, marked improvement but occasional pain; fair, some improvement with significant functional restrictions and the need for pain medications; and poor, no change in or worsening of symptoms. ${ }^{17}$ Following minimally invasive resection of their synovial cysts, 10 patients had excellent results, eight had good results, and one had a fair result. The one patient with a fair result noticed an improvement in her radicular symptoms but continued to have low-back pain that required pain medication. Flexion-extension films showed no gross instability, and she subsequently improved with physical therapy. 

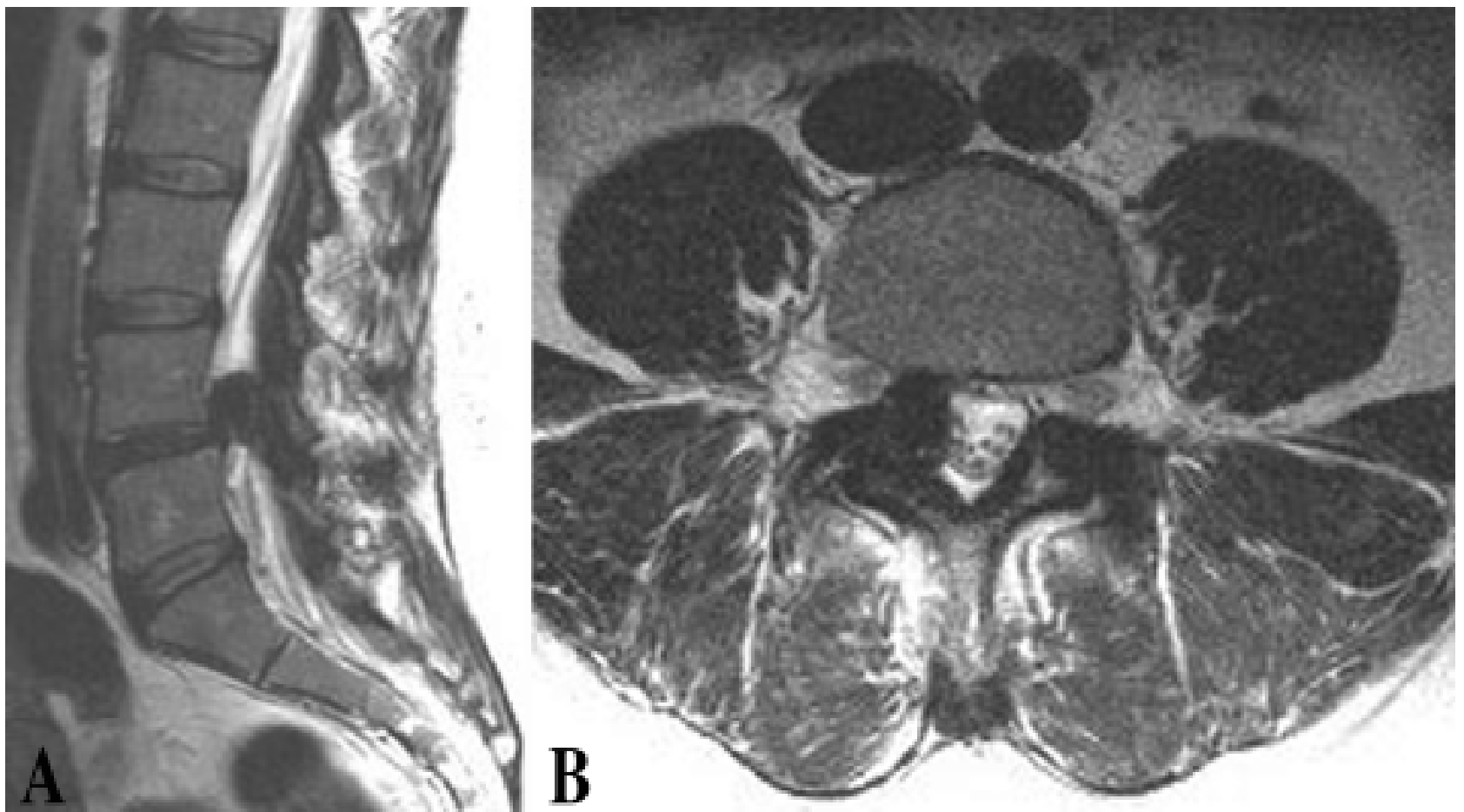

FIG. 1. Sagittal (A) and axial (B) $\mathrm{T}_{2}$-weighted MR images demonstrating a large right-sided L4-5 synovial cyst.

The average duration of surgery was 158 minutes (range 75-270 minutes). The mean intraoperative EBL was $31 \mathrm{ml}$ (range 10-100 $\mathrm{ml}$ ). The only complications were the two previously mentioned intraoperative dural tears, which were repaired with primary dural closure and placement of fat grafts. Neither of these patients had any short- or long-term sequelae as a result of this complication.

\section{Discussion}

Overview of Synovial Cysts

The exact cause of lumbar synovial cysts remains unclear; however, it is widely accepted that their incidence is largely related to advanced degeneration of the facet joint. This is supported by the proximity of the cysts to the de-

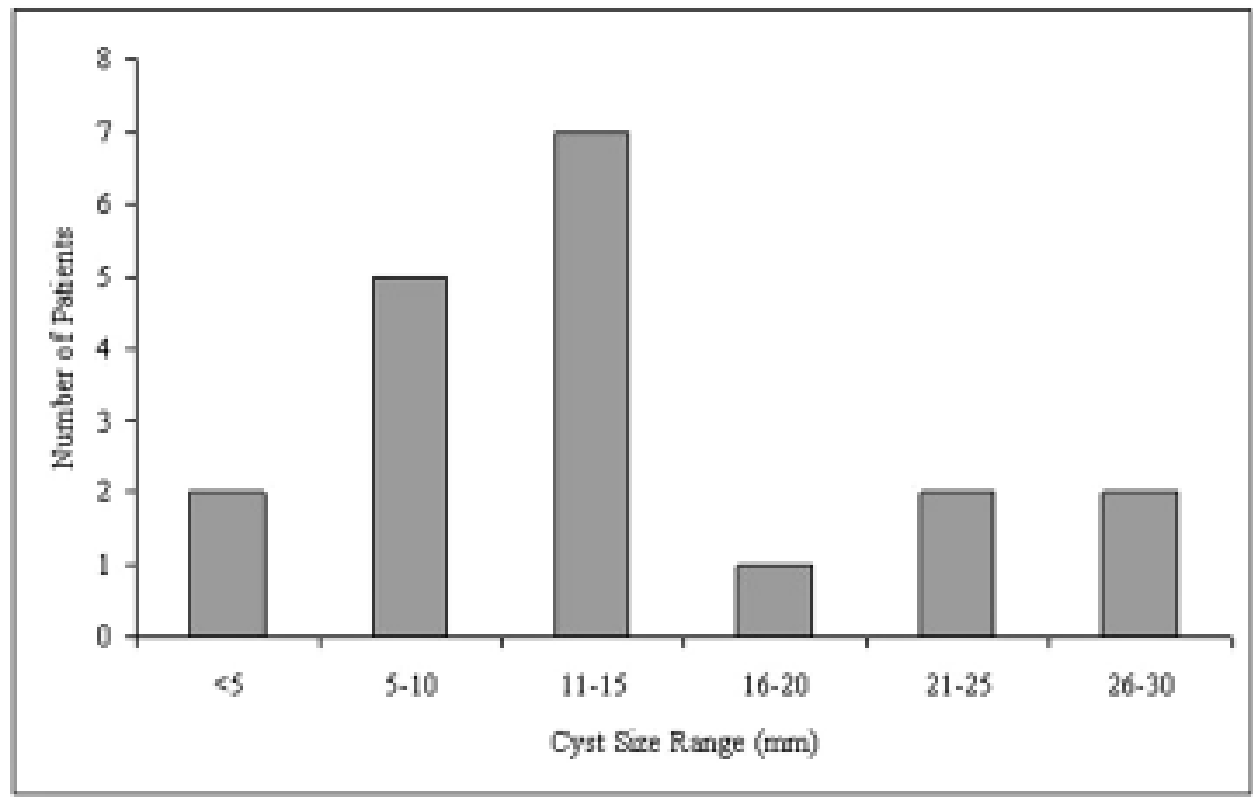

FIG. 2. Bar graph showing the wide variation of cyst size within the cohort. 


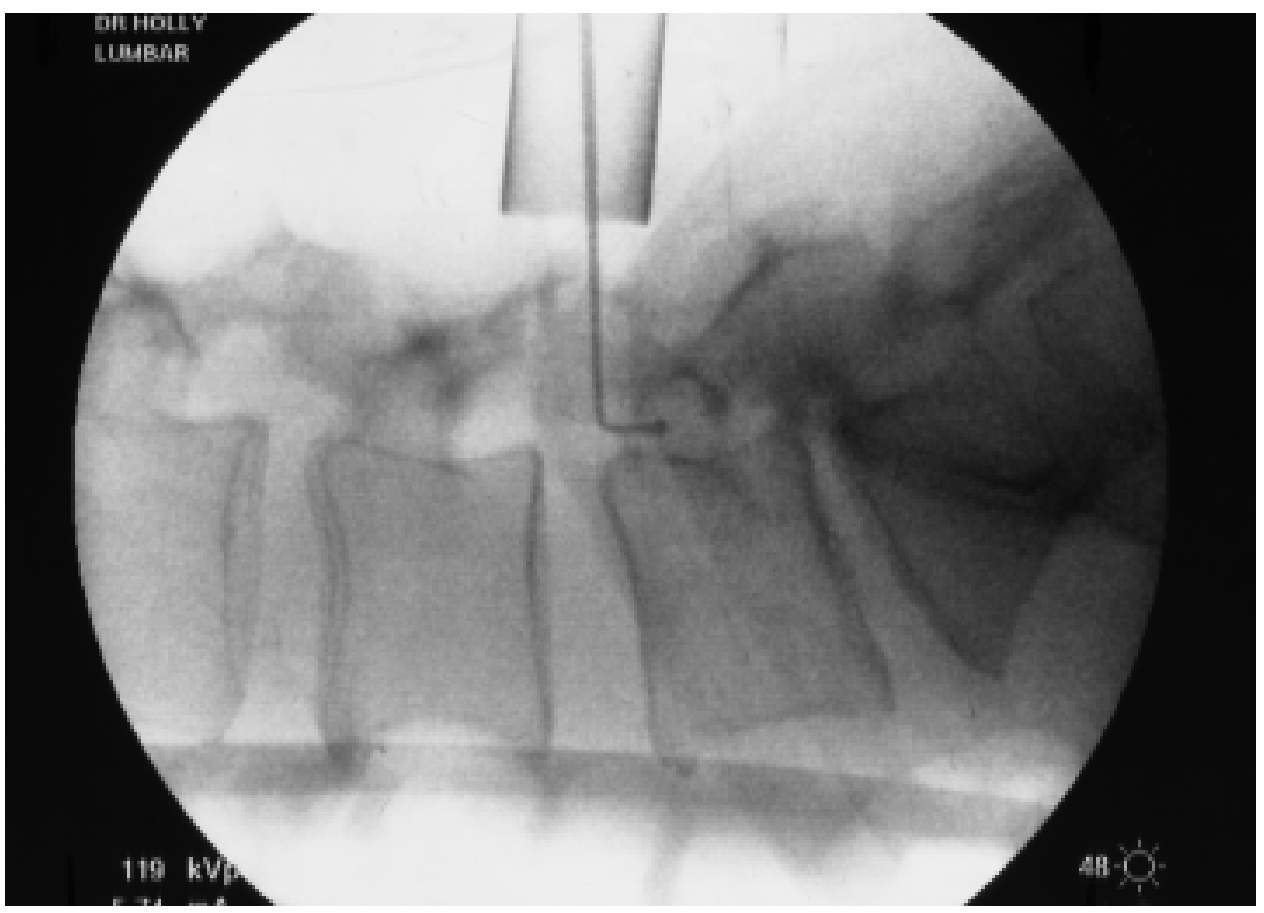

FIG. 3. Intraoperative fluoroscopic image obtained during minimally invasive L4-5 synovial cyst resection. The ball hook is being used to identify the location of the pedicle, which serves as an important anatomical landmark.

generated joints and the fact that these cysts tend to occur in older individuals. As with other manifestations of degenerative disease, it is likely that segmental instability and trauma may also play a role in the pathogenesis of the disease. Synovial cysts can also be observed in patients with systemic inflammatory conditions such as rheumatoid arthritis. ${ }^{6}$

The actual incidence of lumbar synovial cysts is unknown because the vast majority of patients harboring these cysts are likely to be asymptomatic. However, the cysts can become symptomatic due to mass effect on adjacent neural structures. The osseous confines of the lateral recess often magnify the degree of impingement, and even smaller cysts can cause significant symptoms (Fig. 4). Consequently, the most common presentation is radicular pain and/or abnormalities in motor or sensory function. Kao, et al., ${ }^{7}$ provided one of the first reports of symptomatic lumbar synovial cysts in a series of three elderly patients with nerve root pain caused by these lesions.

\section{Management of Lumbar Synovial Cysts}

A variety of nonoperative management techniques have been advocated for the treatment of symptomatic synovial cysts. $2,9,10,11,15,18$ Standard methods such as physical therapy, epidural and oral steroid drugs, and nonsteroidal antiinflammatory drugs are usually the first line of treatment in patients without neurological deficit. Percutaneous treatment strategies, which are less commonly used, include injection of steroid drugs into the facet joint and direct aspiration of the synovial cyst. Parlier-Cuau, et al. ${ }^{15}$ reported a series of 30 patients with nerve root pain caused by a synovial cyst who underwent facet joint steroid injection. They found that only 10 of the patients had satisfactory pain relief at 6-month follow up. Shah and Lutz ${ }^{18}$ per- formed cyst aspiration followed by intracystal steroid injection in five patients, and five patients had cyst aspiration followed by epidural steroid injection. The average follow up was 50 weeks, and only one patient had sustained relief. Eight of the nine patients with continued pain underwent surgery, and all of these patients then experienced pain relief.

Excision of the synovial cyst is highly effective for pain relief and appears to be the definitive treatment for these

TABLE 2

Patient outcome and cyst size

\begin{tabular}{rrlrrr}
\hline \hline $\begin{array}{c}\text { Case } \\
\text { No. }\end{array}$ & $\begin{array}{c}\text { Cyst } \\
\text { Size }(\mathrm{mm})\end{array}$ & Outcome* & EBL $(\mathrm{ml})$ & Op (mins) & $\begin{array}{c}\text { LOS } \\
\text { (days) }\end{array}$ \\
\hline 1 & 10 & excellent & 10 & 151 & 0 \\
2 & 8 & good & 10 & 172 & 2 \\
3 & 25 & excellent & 10 & 96 & 0 \\
4 & 12 & excellent & 10 & 144 & 0 \\
5 & 22 & excellent & 10 & 78 & 0 \\
6 & 30 & good & 100 & 177 & 6 \\
7 & 14 & excellent & 50 & 140 & 3 \\
8 & 28 & good & 10 & 75 & 0 \\
9 & 15 & good & 10 & 83 & 1 \\
10 & 10 & excellent & 50 & 212 & 0 \\
11 & 12 & excellent & 25 & 228 & 0 \\
12 & 7 & good & 75 & 159 & 0 \\
13 & 3 & good & 25 & 170 & 0 \\
14 & 3 & excellent & 25 & 156 & 0 \\
15 & 9 & good & 30 & 120 & 0 \\
16 & 15 & good & 30 & 153 & 0 \\
17 & 7 & excellent & 30 & 202 & 0 \\
18 & 14 & excellent & 50 & 238 & 1 \\
19 & 18 & fair & 25 & 270 & 2 \\
\hline
\end{tabular}

* Based on the Macnab modified criteria. 


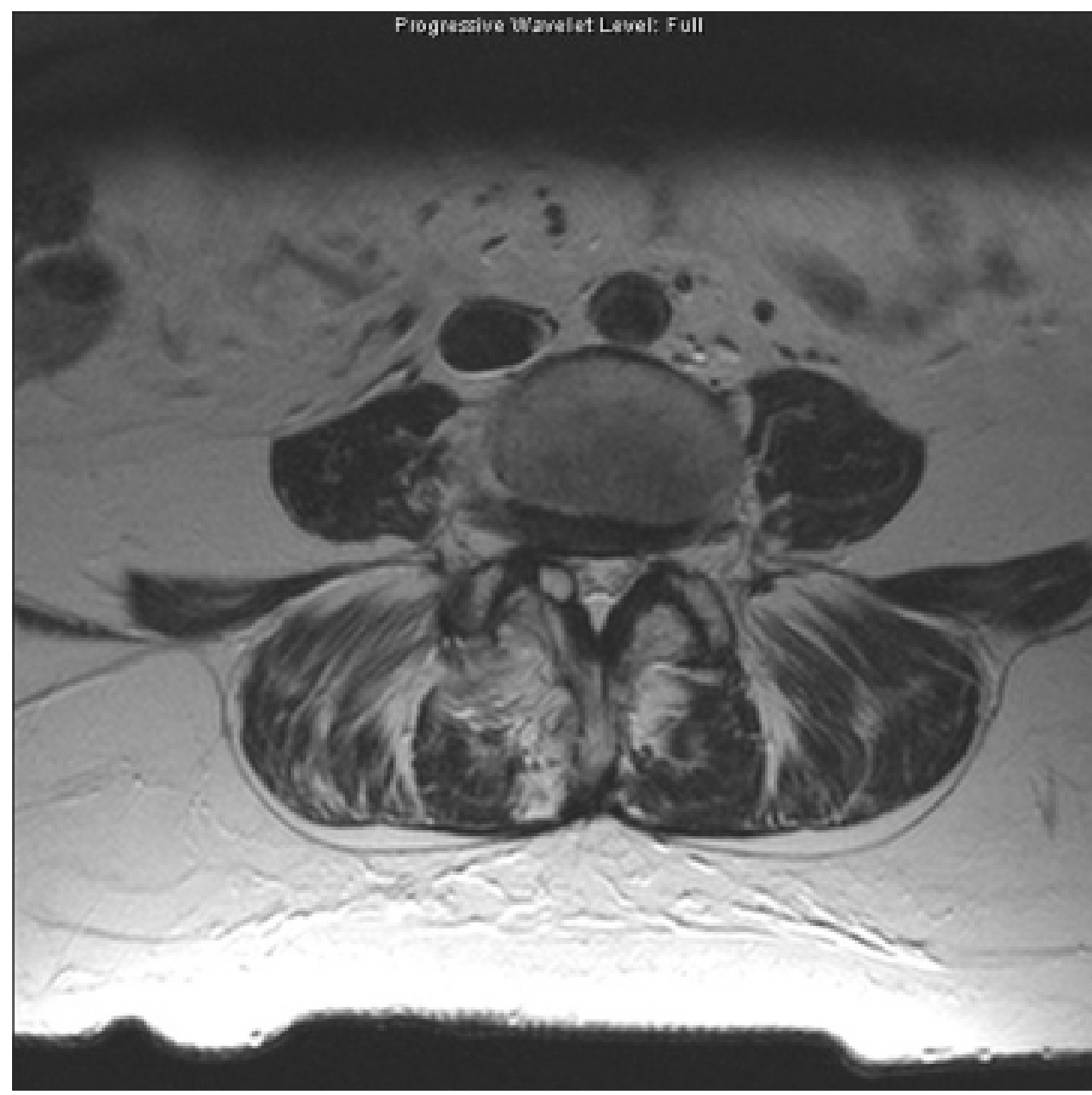

FIg. 4. Axial MR image demonstrating a small symptomatic cyst located in the right lateral recess.

lesions. The vast majority of the reports in the medical literature describe cyst resection via a standard open approach. ${ }^{1,5,8,12,14,16}$ Lyons, et al., ${ }^{12}$ reported the results of 194 patients who underwent surgery for symptomatic synovial cysts with a minimum of 6 months of follow up. Eighteen patients required a concomitant fusion because they showed evidence of preoperative instability, and subsequent fusion was required in four other patients because of postoperative instability. Ninety-one percent of the patients reported good pain relief, and $82 \%$ experienced improvement in motor strength. Sabo, et al., ${ }^{16}$ described a series of 53 patients who underwent synovial cyst resection with a mean follow up of 12 months. Six of these patients underwent fusion for preoperative instability, and two patients later underwent fusion for development of instability postoperatively.

There has been one previous investigation describing the minimally invasive resection of lumbar synovial cysts. Sandhu, et al., ${ }^{17}$ reported a series of 17 patients with a mean follow up of 13 months. Excellent or good results were obtained in 16 patients (94\%). In our study, 18 (95\%) of 19 patients obtained either excellent or good clinical outcomes, and there were no cases of delayed instability within the 16-month follow-up period. The majority of our patients were discharged home the same day of surgery, and the mean EBL was $31 \mathrm{ml}$. The amount of blood loss was very similar to that reported by Sandhu, et al., and significantly less than that attributed to conventional synovial cyst resection.

In addition to the shortened LOS and decreased intraoperative blood loss, one of the other potential benefits of the minimally invasive technique is the preservation of the dorsal muscular and ligamentous attachments. These structures play an important role in the maintenance of spinal stability, and we believe that any effort to preserve their integrity is a worthwhile undertaking. Even in situations in which a laminectomy is required for decompression, minimally invasive techniques allow this procedure to be performed with relatively little disruption of the midline muscular and ligamentous structures. Although the follow-up duration was relatively short in both of the aforementioned investigations of minimally invasive synovial cyst resection, there was no evidence of delayed instability following the decompression. Larger study groups and longer follow-up periods will be needed to determine whether the minimally invasive approach can actually decrease the likelihood that a subset of such patients will require fusion. 


\section{Conclusions}

Resection is the definitive treatment for symptomatic lumbar synovial cysts. While the traditional open procedure for resection of the cyst has been associated with satisfactory results, the recent advent of minimally invasive surgical techniques has significantly decreased the amount of intraoperative blood loss and LOS. Further investigation is necessary to determine whether this novel technique can reduce the need for fusion in some patients.

\section{References}

1. Banning CS, Thorell WE, Leibrock LG: Patient outcome after resection of lumbar juxtafacet cysts. Spine 26:969-972, 2001

2. Bureau NJ, Kaplan PA, Dussault RG: Lumbar facet joint synovial cyst: percutaneous treatment with steroid injections and distention-clinical and imaging follow-up in 12 patients. Radiology 221:179-185, 2001

3. Foley KT, Holly LT, Schwender JD: Minimally invasive lumbar fusion. Spine 28:S26-S35, 2003

4. Foley KT, Smith MM: Microendoscopic discectomy. Tech Neurosurg 3:301-307, 1997

5. Howington JU, Connolly ES, Voorhies RM: Intraspinal synovial cysts: 10-year experience at the Ochsner Clinic. J Neurosurg 91:193-199, 1999

6. Jacob J, Weisman MH, Mink JH, et al: Reversible cause of back pain and sciatica in rheumatoid arthritis: an apophyseal joint cyst. Arthritis Rheum 29:431-435, 1986

7. Kao CC, Uihlein A, Bickel WH, et al: Lumbar intradural extradural ganglion cyst. J Neurosurg 29:168-172, 1968

8. Khan AM, Synnot K, Cammisa FP, et al: Lumbar synovial cysts of the spine: an evaluation of surgical outcome. J Spinal Disord Tech 18:127-131, 2005

9. Koenigsberg RA: Percutaneous aspiration of lumbar synovial cyst: CT and MRI considerations. Neuroradiology 40:272-273, 1998
10. Lim AK, Higgins SJ, Saifuddin A, et al: Symptomatic lumbar synovial cyst: management with direct CT-guided puncture and steroid injection. Clin Radiol 56:990-993, 2001

11. Lutz GE, Shen TC: Fluoroscopically guided aspiration of a symptomatic lumbar zygapophyseal joint cyst: a case report. Arch Phys Med Rehabil 83:1789-1791, 2002

12. Lyons MK, Atkinson JL, Wharen RE, et al: Surgical evaluation and management of lumbar synovial cysts: the Mayo Clinic experience. J Neurosurg 93:53-57, 2000

13. Macnab I: Negative disc exploration. An analysis of the causes of nerve root involvement in sixty-eight patients. J Bone Joint Surg Am 53:891-903, 1971

14. Onofrio BM, Mih AD: Synovial cysts of the spine. Neurosurgery 22:642-647, 1988

15. Parlier-Cuau C, Wybier M, Nizard R, et al: Symptomatic lumbar facet joint synovial cysts: clinical assessment of facet joint steroid injection after 1 and 6 months and long-term follow-up in 30 patients. Radiology 210:509-513, 1999

16. Sabo RA, Tracy PT, Weinger JM: A series of 60 juxtafacet cysts: clinical presentation, the role of spinal instability, and treatment. J Neurosurg 85:560-565, 1996

17. Sandhu FA, Santiago P, Fessler RG, et al: Minimally invasive surgical treatment of lumbar synovial cysts. Neurosurgery 54: $107-112,2004$

18. Shah RV, Lutz GE: Lumbar intraspinal synovial cysts: conservative management and review of the world's literature. Spine J 3:479-488, 2003

Manuscript received January 17, 2006.

Accepted in final form February 24, 2006.

Address reprint requests to: Langston T. Holly, M.D., Division of Neurosurgery, 74-144 CHS, 10833 Le Conte Avenue, David Geffen University of California at Los Angeles School of Medicine, Los Angeles, California 90095. email: 1holly@ mednet.ucla.edu. 